\title{
Dynamique du couvert végétal à Bassila au nord Bénin pendant et après la mise en œuvre d'un projet d'aménagement forestier
}

\author{
Oméga Lydia Clarisse GBEDAHI ${ }^{*}$, Samadori Sorotori Honoré BIAOU ${ }^{1}$, Adi MAMA ${ }^{2}$, \\ Gérard Nounagnon GOUWAKINNOU ${ }^{1}$ et Nourou Soulémane YOROU ${ }^{1}$ \\ ${ }^{1}$ Laboratoire d'Ecologie, de Botanique et de Biologie végétale (LEB), Faculté d'Agronomie (FA), Université \\ de Parakou (UP), O3BP 125, Parakou, Bénin. \\ ${ }^{2}$ Laboratoire d'Ecologie Appliquée (LEA), Faculté des Sciences Agronomiques(FSA), Université d'Abomey \\ Calavi (UAC), 01 BP 1651 Abomey-Calavi, Bénin. \\ *Auteur correspondant ; E-mail: lydiagbedahi@gmail.com, Tél : (+229) 6620264 /95620565
}

\section{RESUME}

Dans un contexte de récurrence de la déforestation, la présente étude a eu pour objectif d'évaluer l'impact du Projet de Restauration des Ressources Forestières de Bassila (PRRF) sur la dynamique du couvert végétal, sur une période de trente ans (1986-2017) dans la commune de Bassila au nord du Bénin. La question de recherche posée est : la mise en œuvre du PRRF a-t-elle favorisé la conservation des forêts de Bassila ? La télédétection et les Systèmes d'informations géographiques ont servi à évaluer la dynamique du couvert végétal pendant projet (1986-2003) et après projet (2003-2017). Entre 1986 et 2017, les superficies forestières dans la zone d'intervention du PRRF ont faiblement augmenté (5,1\% à 6\%). Mais, on remarque qu'en réalité, les forêts ont d'abord doublé de superficie pendant la mise en œuvre du PRRF (5,1\% à 11\%) pour, ensuite, disparaitre de moitié après la fin du PRRF (11\% à 6\%). Bien que la mise en œuvre du PRRF ait favorisé la restauration des forêts dans la commune de Bassila, sa fin semble avoir été défavorable à la conservation de ces forêts. Un suivi des activités d'aménagement, après la fin des projets, est donc recommandé pour la gestion durable des ressources forestières.

(C) 2019 International Formulae Group. All rights reserved.

Mots clés: Forêts, projets forestiers, dégradation, restauration, Bassila, République du Bénin.

\section{Dynamic of vegetation cover in Bassila in northern Benin before and after a forest management project}

\begin{abstract}
In a context of recurrence of deforestation, the objective of this study was to assess the impact of the Bassila Forest Resource Restoration Project (PRRF) on vegetation cover dynamics over a 30 year period (1986-2017) in the Commune of Bassila in the north of Benin. The research question addressed is: has the implementation of PRRF favored the conservation of the Bassila forest resources? Remote sensing and Geographic Information Systems (GIS) were used to evaluate vegetation cover dynamics during the project (1986-2003) and after the project (2003-2017). Between 1986 and 2017, the forest areas in the PRRF intervention area increased slightly (5.1\% to 6\%). Nevertheless, we notice that in fact, the forests first doubled
\end{abstract}


in size during the implementation of the PRRF (5.1\% to $11 \%$ ) and then disappeared by half after the end of the PRRF (11\% to $6 \%$ ). Although the project implementation promoted the restoration of forest resources in the Commune of Bassila, its end seems to have been unfavorable to the conservation of these resources. Therefore, it is necessary to develop follow-up conservation efforts for the sustainable management of forest resources.

(C) 2019 International Formulae Group. All rights reserved.

Keywords: Forests, forestry projects, degradation, restoration, Bassila, Republic of Benin.

\section{INTRODUCTION}

La majorité des trente-trois (33) pays, dans le monde, ayant connu, entre 2000 et 2010, une perte nette d'espace forestiers et un gain net d'espaces agricoles se trouvent en Afrique (FAO, 2016). La déforestation, la fragmentation (Ngueguim et al., 2009) la savanisation sont autant de conséquences de l'anthropisation des espaces forestiers tropicaux (Geist et Lambin, 2001). Il en ressort, entre autres, une grande perte de la biodiversité et des services écosystémiques, ainsi que la raréfaction des ressources forestières, avec des répercussions sociales et économiques énormes. Les forêts aménagées et gérées avec l'appui de projets forestiers ne sont pas épargnées. Dans bien des cas, ces forêts aménagées et gérées par l'Etat par le biais de projets forestiers ont connu une dégradation quasi irrémédiable (Atindogbé, 2004), ternissant le blason de la gestion participative dont elles font l'objet (Vermeulen et al., 2006). Au Bénin, la déforestation et la dégradation des forêts serait due à la forte pression découlant des activités anthropiques dans les formations végétales (Atindogbé, 2004; Mama et al., 2014 ; Avakoudjo et al., 2014). Néanmoins, la grande majorité des études portant sur la dynamique de la végétation au Bénin ont été effectuées à l'échelle de vastes territoires géographiques et peu d'études se focalisent spécifiquement sur les zones d'intervention de projets forestiers. Ces études ne permettent donc pas d'évaluer le succès des projets forestiers quant à la conservation et au développement des ressources forestières dans leur zone d'intervention. L'un de ces projets est le Projet de Restauration des Ressources Forestières de Bassila (PRRF).
Le PRRF est, au Bénin, l'un des tous premiers projets d'aménagement forestier, initié en 1988 pour pallier les retombées négatives de l'écrémage excessif des forêts de la commune de Bassila (PRRF, 2003). Il a connu cinq (5) phases dont la dernière a pris fin en 2004. Toute la commune de Bassila, sous-préfecture de Bassila d'alors, excepté les massifs forestiers des Monts Kouffè et de Wari-Maro (PRRF, 2003) a constitué la zone d'intervention du PRRF. Du fait de la période de mise en œuvre relativement longue du PRRF, la Commune de Bassila est un excellent cas d'étude pour vérifier, au Bénin, la capacité des projets d'aménagement forestiers à stopper la dégradation des forêts et à permettre une gestion durable des ressources forestières. L'objectif du présent travail est d'évaluer la dynamique d'occupation des sols dans la zone d'intervention du PRRF depuis sa mise en œuvre jusqu'en 2017 (30 ans), en mettant en exergue les différences entres les périodes pendant projet (1986-2003) et après projet (2003-2017). Les questions spécifiques suivantes ont été adressées : (i) Le PRRF a-t-il favorisé la restauration des ressources forestières dans la commune de Bassila? (ii) Si oui, cet impact est-il prolongé au-delà de la période d'intervention du projet ?

\section{MATERIEL ET METHODES Milieu d'étude}

Cette étude a été conduite dans la zone d'intervention du PRRF (Figure 1) qui couvre toute la commune de Bassila à l'exception des forêts classées des Monts Kouffè et de WariMaro (PRRF, 2003). Cette zone est comprise entre $8^{\circ} 31$ et $9^{\circ} 29$ latitude Nord et entre $1^{\circ} 22$ et $2^{\circ} 15$ longitude Est et s'étend sur une superficie totale de 250000 ha (PRRF, 2003). 
Le climat y est de type soudano-guinéen avec une moyenne pluviométrique de $1200 \mathrm{~mm}$ par an répartis sur six (6) à sept (7) mois (Houinato et al., 2013). L'humidité relative oscille entre $15 \%$ (janvier) en saison sèche et 99\% (aout - septembre) en saison pluvieuse (PRRF, 2003). Les sols sont principalement de type ferrugineux sableux, ferralitiques et hydromorphes (Hincourt, 1991). Le réseau hydrographique est assez dense et constitué de nombreux ruisseaux et petits cours d'eau (PRRF, 2003).

\section{Collecte de données}

La collecte des données s'est effectuée à partir de trois scènes de l'imagerie Landsat suivant les années de début (1988) et de fin (2003) du PRRF et l'année de déroulement de l'étude (2017). Mais notons que dans le cadre de la présente étude, une image de 1986 a été utilisée en lieu et place de celle de 1988 à cause de l'indisponibilité d'image de 1988 de bonne qualité. Ainsi deux images Landsat 7 (l'une de Janvier 1986 et l'autre d'Avril 2003) et une image Landsat 8 (de Janvier 2017) ont été utilisées, ayant une résolution spatiale de $30 \mathrm{~m} \times 30 \mathrm{~m}$ et correspondant toutes à la feuille path 192 et row 054. Elles présentent l'avantage d'être acquises gratuitement sur le site de l'USGS. Nous avons aussi utilisé la carte topographique (NC-31-II, NC-31-III, NB-31-XX-XXI) au 1/200000 du Bénin (IGN France, 1969), les vérifications terrain et le logiciel Google Earth Pro pour mieux discriminer les classes d'occupation du sol.

Par ailleurs, pour évaluer l'impact de la cessation du PRRF sur les activités d'aménagement, des personnes ressources telles que des agents forestiers, des élus locaux et d'anciens membres des comités locaux de gestion des forêts aménagées ont fait l'objet d'une enquête informelle.

\section{Traitement des images satellites}

Le traitement et l'analyse des images satellites ont été faits avec les logiciels ENVI 4.5 (images Landsat 7) et Multispec (image Landsat 8) pour la classification, et QGIS
2.14.0 et ArcGIS 9.3.1 pour les analyses spatiales.

Le traitement des images a consisté essentiellement à effectuer une classification supervisée des images puis à cartographier cinq (05) classes d'occupation du sol que sont : "Forêts", "Savanes", "ChampsJachères", "Sols nus et agglomérations" et "plantation arborées". Pour cela, les filtres de Kernel avec des fenêtres de 7 X 7 pixels ont été appliqués aux images classifiées afin de résorber les pixels isolés

Un contrôle de terrain a été effectué de Décembre 2016 à Janvier 2017 et a consisté à vérifier en milieu réel deux cents (200) points précédemment pris sur la classification et encodés dans un GPS.

\section{Evaluation de la précision des classifications}

Pour chacune des trois images traitées, la précision de la classification a été estimée à partir des matrices de confusion. Les mesures utilisées étaient la précision globale, le coefficient de Kappa, les erreurs de commission et d'omission (Foody, 2002 ; Abdel-Kawy et al., 2011).

\section{Mise en évidence des changements d'occupation de sol}

Les cartes d'occupation des sols obtenues après classification ont été superposées deux à deux afin d'obtenir les cartes de changements du paysage de la zone d'étude. Nous avons procédé par la suite à la création des matrices de transition pour faire ressortir les fréquences de transition entre les classes (Robin, 2002 ; Barima et al., 2009) de 1986 à 2003 (pendant projet), de 2003 à 2017 (après projet) et de 1986 à 2017 (toute la période d'observation).

La caractérisation de la structure spatiale du paysage s'est appuyée sur le nombre de taches (n), la superficie (s) et le périmètre cumulatif des taches ( $p$ ) dans chaque classe d'occupation du sol. L'arbre de décision de Bogaert et al. (2004) nous a permis de déterminer les processus majeurs de transformation spatiale (PTS) du paysage. Lorsque le nombre de taches final est inférieur 
au nombre de taches initial tandis que la superficie finale est inférieure à la superficie initiale, le PTS correspond à la suppression des taches. Dans les cas où le nombre de taches final est supérieur au nombre de taches initial, le rapport $t_{\text {observe }}=$ superficie finale $/$ superficie initiale est comparé à une valeur prédéfinie de $\mathrm{t}=0,5$ afin de distinguer la fragmentation et la dissection. Lorsque $t_{\text {observé }}$ $\leq 0,5$ le PTS est la fragmentation alors qu'une valeur de $t_{\text {observé }}>0,5$ correspond à un PTS de dissection. Le PTS de la création des taches correspond au cas où le nombre de taches final est supérieur au nombre de taches initial et que la superficie finale est supérieure à la superficie initiale. Enfin, on conclut d'un PTS d'agrégation des taches lorsque le nombre de taches final est inférieur au nombre de taches initial tandis que la superficie finale est supérieure à la superficie initiale.

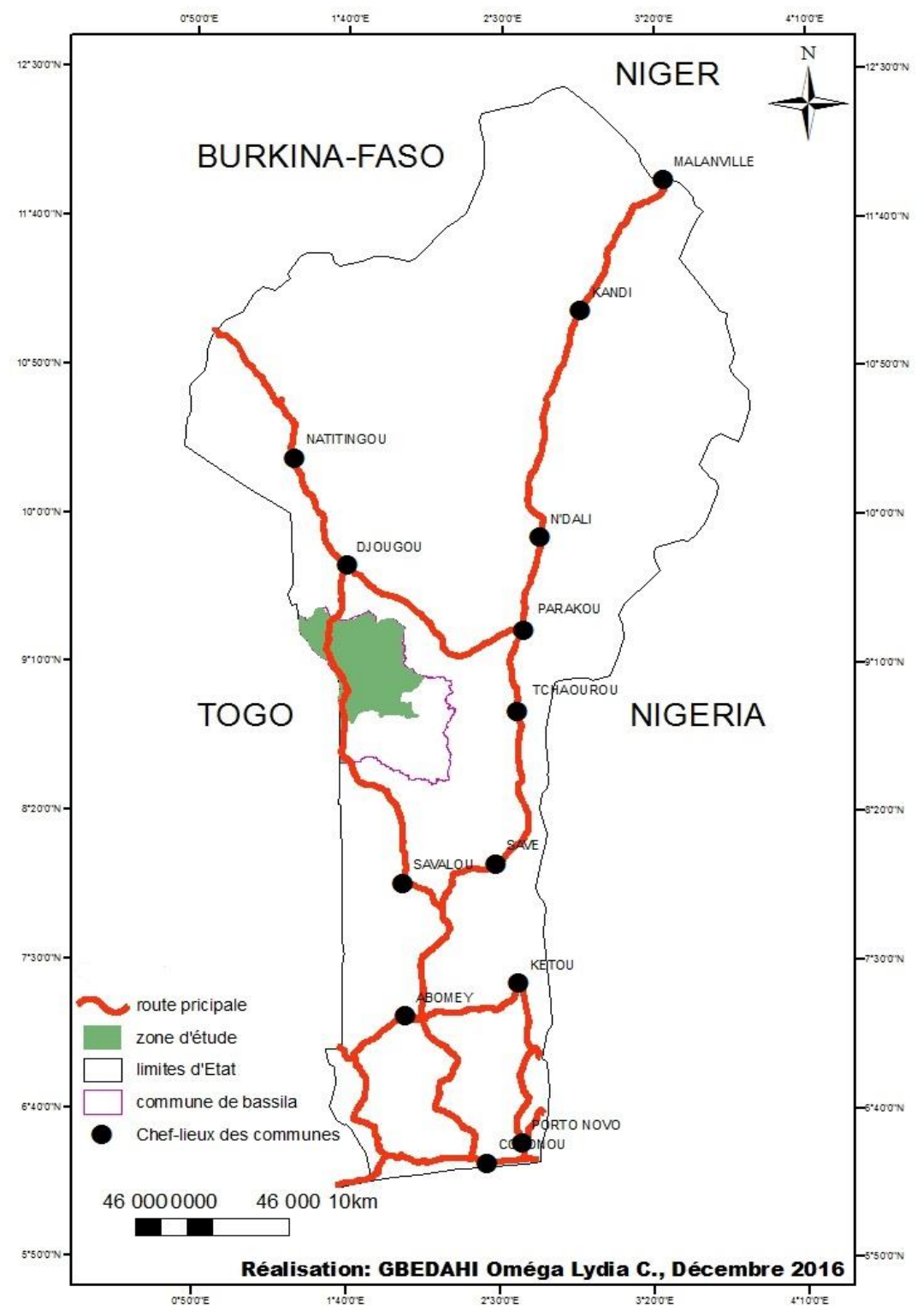

Figure 1: Localisation de la zone d'étude au Bénin. 


\section{RESULTATS}

\section{Exactitude des classifications}

La classification de l'image de 1986 était exacte à $88,8 \%$ (coefficient de Kappa) avec une précision globale de 93,1\% (Tableau 1). Les faibles erreurs de commission et d'omission pour les classes des forêts $(0 \%$ et $12,2 \%$ respectivement) et des savanes $(9,1 \%$ et $0 \%$ respectivement) font de ces deux classes, les moins affectées par les autres classes. De même, la classification de l'image de 2003 avait un coefficient de Kappa de $88,6 \%$, une précision globale de $91,6 \%$, des erreurs de commission et d'omission de $11,97 \%$ et $11,43 \%$ respectivement pour la classe forêt et de $1,24 \%$ et $11,80 \%$ respectivement pour la classe savane. Enfin, la matrice de confusion de l'image de 2017 révèle un excellent coefficient de Kappa $(94,4 \%)$ et une précision globale de $96,0 \%$. Les erreurs de commission des classes forêt et savane (respectivement $1 \%$ et $3,4 \%$ ) et d'omission pour les mêmes classes (respectivement $0,11 \%$ et $0,88 \%$ ) désignent, tout comme pour l'image classifiée de 1986, ces deux classes comme les moins affectées par les autres classes.

\section{Dynamique d'occupation des sols dans la zone d'intervention du PRRF de 1986 à 2017}

En 1986 (Figure 2), la zone d'intervention du PRRF était essentiellement recouverte de formations savanicoles $(90,7 \%$ de la superficie totale), suivies des forêts $(5,1 \%)$, des champs et jachère $(2,6 \%)$ et des plantations $(1,4 \%)$ alors que les sols nus et agglomérations venaient en dernière position $(0,2 \%)$.

De 1986 à 2017, les formations savanicoles ont connu une régression spatiale $(90,7$ à $73,2 \%$ de la superficie totale $)$ contrairement aux plantations et champs et jachères qui se sont étendues davantage $(1,4 \%$ à $8,8 \%$ pour les plantations et $2,6 \%$ à $11,5 \%$ pour les champs et jachères). Les forêts $(5,1 \%$ à $6 \%)$ et les sols nus et agglomérations $(0,2 \%$ à $0,5 \%$ ) ont aussi globalement augmenté en superficies de 1986 à 2017 bien qu'en plus faibles proportions.

\section{Transitions entre les classes d'occupation des sols de 1986 à 2017 dans la zone d'intervention du PRRF}

Dans la période allant de 1986 à 2017, les formations savanicoles bien que demeurant la matrice dominante du paysage en 2017, sont passées de $90,7 \%$ à 73,2\% (Tableau 2). Cette régression des savanes s'est faite essentiellement au profit des champs et jachères et des plantations arborées. En effet, les champs et jachères sont passés de 2,6\% de la superficie totale en 1986 à $11,5 \%$ en 2017 grâce à une reconversion de $9,9 \%$ des savanes contre seulement $0,5 \%$ de reconversion des forêts et $0,2 \%$ de reconversion des plantations. Les plantations arborées quant-à-elles sont passées de $1,4 \%$ à $8,8 \%$ grâce à une reconversion de $8 \%$ de savanes, $0,2 \%$ des forêts et $0,4 \%$ de champs et jachères. Notons qu'il s'agit essentiellement de plantations monospécifiques de teck, d'anacardiers et de Gmelina. Les forêts passent seulement de $5,1 \%$ à $6 \%$, soit un accroissement d'à peine $0,9 \%$ sur les trente (30) années d'observation. Plus de la moitié $(2,8 \%)$ des $5,1 \%$ de surface totale de la zone occupés par les forêts en 1986 a entièrement disparu en 2017. La faible augmentation des superficies de forêts $(5,1 \%$ à $6 \%)$ observée est en réalité due à l'apparition de nouveaux espaces forestiers provenant de la reconversion de $3,6 \%$ de savanes et $0,1 \%$ de plantations. Enfin, la classe sol nu et agglomération a évolué de $0,2 \%$ à $0,5 \%$ par la destruction de $0,3 \%$ de savanes.

La matrice de transition des classes d'occupation des sols de 1986 à 2003 (période de mise en œuvre du projet) et celle de 2003 à 2017 (période après projet) nous renseigne sur le fait que les forêts ont dans un premier temps doublé de superficie $(5,1 \%$ à $11 \%)$ pendant la période de mise en œuvre du projet (Tableau 2) et ce en grande partie au détriment des savanes $(7,1 \%$ des $90,7 \%$ initiaux de savanes reconvertis en forêt) avant de disparaitre à moitié (11\% à 6\%) après la fin du projet entre 2003 et 2017 par la savanisation de $5,7 \%$ de leurs superficies (Figure 4). Les sols nus et agglomérations qui ont d'abord accru de 0,2\% à 1,4\% (1986 à 2003), ont régressé par la suite en passant à $0,5 \%$ de la superficie au profit des champs et jachères et des savanes. Parallèlement, les 
savanes ont perdu $7,1 \%$ de leurs superficies en forêts contre respectivement $6,2 \%$ et $8,8 \%$ en plantation arborées et en champs et jachères entre 1986 et 2003 d'une part et plutôt 1,8\%, $6,2 \%$ et $8,8 \%$ de leurs superficies respectivement en forêts, en plantations arborées et en champs et jachères entre 2003 et 2017 d'autre part.

\section{Dynamique de la structure spatiale}

De 1986 à 2003, il est observé une augmentation simultanée du nombre de taches (n), de la superficie (s) et du périmètre (p) pour les plantations arborées, les sols nus et agglomérations et les forêts (Tableau 3). Il est clair que le PTS qui a prévalu pour ces trois classes d'occupation de sol durant cette période est la création (Figure 3). Par contre pour les champs et jachères, il est plutôt observé une diminution du nombre de taches et une augmentation des surfaces et du périmètre cumulatif. Ceci nous permet de conclure à un PTS d'agrégation pour cette classe d'occupation du sol. Quant aux savanes, l'augmentation simultanée du nombre de taches et du périmètre cumulatif est accompagnée d'une diminution des superficies occupées. La valeur de t observé $(0,89)$ nous indique pour les savanes un PTS de dissection.

Durant la période allant de 2003 à 2017, seuls les champs et jachères ont été sujet à un processus de création alors que les sols nus et agglomération ont subi une suppression de leurs taches (Tableau 3). Le processus d'agrégation (fusion des taches) a concerné la classe des plantations arborées pour lesquelles le nombre de taches a diminué, tandis que la superficie et le périmètre cumulatifs ont augmenté. Les forêts ont subi un processus de suppression (diminutions simultanées du nombre de taches, de la superficie et du périmètre cumulatif) (Figure 4). Enfin, la dissection observée au niveau des formations savanicoles dans la période allant de 1986 à 2003 s'est poursuivie de 2003 à 2017.

En résumé, de 1986 à 2017, la dynamique de la structure spatiale dans la zone d'intervention du PRRF révèle un accroissement régulier des espaces agricoles (création de nouvelles taches de champs et jachères, création et agrandissement des taches de plantation), une dégradation (dissection) des formations savanicoles, une restauration des espaces forestiers (1986 à 2003) suivi d'une déforestation (2003 à 2017) et une suppression des taches de sols nus et agglomérations (2003 à 2017).

\section{Conséquence de la fin du PRRF sur la mise en œuvre des activités de conservation des forêts}

Dans le cas de l'aménagement des massifs forestiers de Bassila, des activités telles que l'entretien des plantations installées avec l'appui du PRRF et leur surveillance incombent aux populations locales par le biais de divers comités locaux de gestion des forêts, montés et installés sous le PRRF. Plusieurs membres de ces comités retrouvés et enquêtés (données non publiées) ont reconnu que ces activités ne sont pratiquement plus menées depuis la fin du projet. Les réunions des comités ne se tiennent plus comme prévu. Un ancien garde-forêt qui était payé par le PRRF pour l'entretien et la surveillance des plantations des forêts communautaires dans l'arrondissement de Bassila a confié qu'il a dû, après le départ de l'équipe de projet, abandonner toute activité puisqu'il n'était plus rémunéré. $\mathrm{Au}$ niveau de quelques massifs forestiers communautaires, comme dans les forêts classées, des habitudes sont restées. Il s'agit notamment de la pratique des feux de brousse précoces et de l'interdiction de couper du bois. Mais là encore, des conflits familiaux ont parfois comme conséquence la dissolution des comités et donc un manque de suivi rigoureux de la gestion des massifs forestiers. Par ailleurs, les activités de sensibilisation de la population à la gestion durable des ressources forestières ont aussi pris fin avec la cessation des activités du projet, à en croire les personnes enquêtées. Ce relâchement dans le suivi des activités d'aménagement a émoussé l'engagement des populations pour la gestion durable des ressources forestières. Elles s'adonnent donc à des pratiques inappropriées à la conservation des ressources forestières telle que l'exploitation frauduleuse du bois. 
Tableau 1: Indices d'évaluation de la précision des classifications supervisées des images de 1986, 2003 et 2017 de la zone d'intervention du PRRF dans la commune de Bassila, Bénin.

\begin{tabular}{|c|c|c|c|}
\hline \multirow{8}{*}{1986} & Classes d'occupation du sol & Erreurs de commission $(\%)$ & Erreurs d'omission (\%) \\
\hline & plantations & 0,6 & 18,6 \\
\hline & forets & 0 & 12,2 \\
\hline & Sols nus et agglomération & 4,3 & 6,0 \\
\hline & champs et jachères & 26,3 & 42,6 \\
\hline & savanes & 9,1 & 0 \\
\hline & & Précision Globale: 93,1\% & \\
\hline & & Coefficient de Kappa: $88,8 \%$ & \\
\hline \multirow{8}{*}{2003} & Classes d'occupation du sol & Erreurs de commission (\%) & Erreurs d'omission (\%) \\
\hline & plantations & 24,31 & 9,5 \\
\hline & forêts & 11,9 & 11,4 \\
\hline & Sols nus et agglomération & 2,9 & 0,5 \\
\hline & champs et jachères & 4,4 & 12,2 \\
\hline & savanes & 1,2 & 11,8 \\
\hline & & Précision Globale: 91,6\% & \\
\hline & & Coefficient de Kappa: $88,6 \%$ & \\
\hline \multirow{8}{*}{2017} & Classes d'occupation du sol & Erreurs de commission(\%) & Erreurs d'omission(\%) \\
\hline & plantations & 4,1 & 25,2 \\
\hline & forêts & 1 & 0,1 \\
\hline & Sols nus et agglomération & 17,3 & 26,2 \\
\hline & champs et jachères & 7,7 & 7,4 \\
\hline & savanes & 3,4 & 0,8 \\
\hline & & Précision Globale: $96 \%$ & \\
\hline & & Coefficient de Kappa: 94,4\% & \\
\hline
\end{tabular}

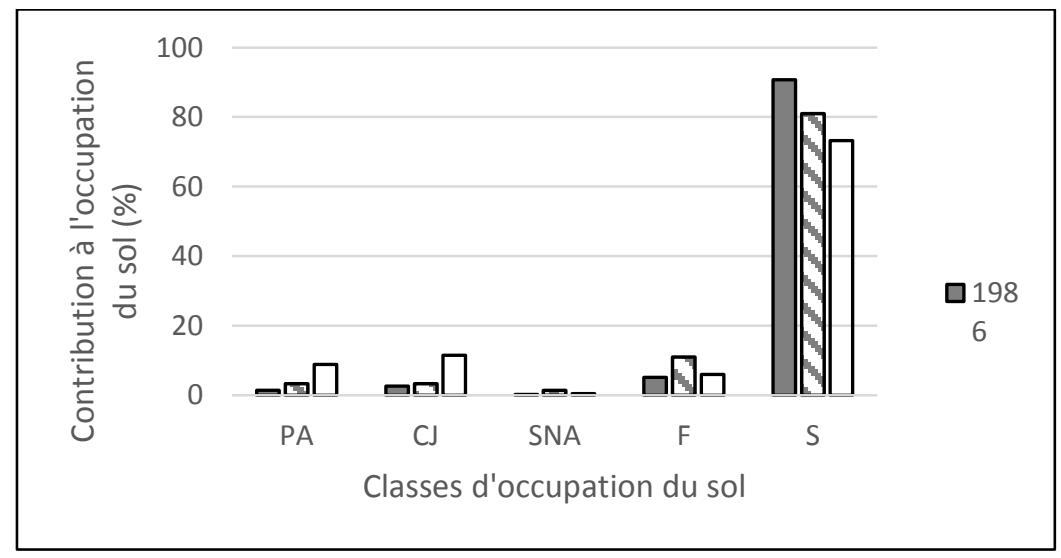

Figure 2: Occupation des sols en 1986, 2003 et 2017 dans la zone d'intervention du PRRF dans la commune de Bassila, Bénin.

Légende $: \mathrm{PA}=$ Plantations, $\mathrm{CJ}=$ Champs et Jachère, $\mathrm{SNA}=$ Sol nu et Agglomération, $\mathrm{F}=\mathrm{Forêt}, \mathrm{S}=\mathrm{Savane}$. 
Tableau 2: Matrice de transition de l'occupation des sols de 1986 à 2003, de 2003 à 2017 et de 1986 à 2017 dans la zone d'intervention du PRRF, commune de Bassila, Bénin.

\begin{tabular}{|c|c|c|c|c|c|c|c|}
\hline & \multicolumn{7}{|c|}{2003} \\
\hline & & PA & $\mathrm{CJ}$ & SNA & $\mathrm{F}$ & $\mathrm{S}$ & Total \\
\hline \multirow{6}{*}{1986} & $\mathrm{PA}$ & 0.3 & 0 & 0 & 0.2 & 0.9 & 1.4 \\
\hline & CJ & 0 & 0.5 & 0.4 & 0.1 & 1.6 & 2.6 \\
\hline & SNA & 0 & 0 & 0.2 & 0 & 0 & 0.2 \\
\hline & $\mathrm{F}$ & 0.4 & 0.1 & 0 & 3.6 & 1 & 5.1 \\
\hline & $\mathrm{S}$ & 2.6 & 2.7 & 0.8 & 7.1 & 77.5 & 90.7 \\
\hline & Total & 3.3 & 3.3 & 1.4 & 11 & 81 & 100 \\
\hline \multirow{10}{*}{2003} & & & & 2017 & & & \\
\hline & & PA & CJ & SNA & $\mathrm{F}$ & $\mathrm{S}$ & Total \\
\hline & $\mathrm{PA}$ & 0.8 & 0.3 & 0 & 0.5 & 1.7 & 3.3 \\
\hline & $\mathrm{CJ}$ & 1 & 1 & 0.1 & 0 & 1.2 & 3.3 \\
\hline & SNA & 0.1 & 0.5 & 0.3 & 0 & 0.5 & 1.4 \\
\hline & $\mathrm{F}$ & 0.7 & 0.9 & 0 & 3.7 & 5.7 & 11 \\
\hline & $\mathrm{S}$ & 6.2 & 8.8 & 0.1 & 1.8 & 64.1 & 81 \\
\hline & Total & 8.8 & 11.5 & 0.5 & 6 & 73.2 & 100 \\
\hline & & & & 2017 & & & \\
\hline & & PA & $\mathrm{CJ}$ & SNA & $\mathrm{F}$ & $\mathrm{S}$ & Total \\
\hline \multirow{6}{*}{1986} & PA & 0.2 & 0.2 & 0 & 0.1 & 0.9 & 1.4 \\
\hline & $\mathrm{CJ}$ & 0.4 & 0.9 & 0 & 0 & 1.3 & 2.6 \\
\hline & SNA & 0 & 0 & 0.2 & 0 & 0 & 0.2 \\
\hline & $\mathrm{F}$ & 0.2 & 0.5 & 0 & 2.3 & 2.1 & 5.1 \\
\hline & $\mathrm{S}$ & 8 & 9.9 & 0.3 & 3.6 & 68.9 & 90.7 \\
\hline & Total & 8.8 & 11.5 & 0.5 & 6 & 73.2 & 100 \\
\hline
\end{tabular}

Légende $: \mathrm{PA}=$ Plantation, $\mathrm{CJ}=$ Champs et Jachère, $\mathrm{SNA}=$ Sol nu et Agglomération, $\mathrm{F}=$ Forêt, $\mathrm{S}=$ Savane .

Tableau 4: Détermination des processus de transformation spatiale des classes d'occupation du sol de 1986 à 2003 et de 2003 à 2017 dans la zone d'intervention du PRRF dans la commune de Bassila, Bénin.

\begin{tabular}{|c|c|c|c|c|c|c|}
\hline Période & COS & n2003 - n1986 & s2003 / s1986 & s1986 - s2003 & p1986 - p2003 & PTS \\
\hline \multirow{6}{*}{1986 - 2003} & PA & 1815 & 2.35 & 5762.61 & 137.99 & Création \\
\hline & $\mathrm{CJ}$ & -34 & 1.37 & 2989.86 & 15.63 & Agrégation \\
\hline & SNA & 996 & 6.37 & 3664.22 & 80.24 & Création \\
\hline & $\mathrm{F}$ & 435 & 2.12 & 17613.64 & 145.93 & Création \\
\hline & $\mathrm{S}$ & 296 & 0.89 & -28271.59 & 283.94 & Dissection \\
\hline & & n2017 - n2003 & s2017/s2003 & s2017-s2003 & p2017-p2003 & \\
\hline \multirow{5}{*}{$2003-2017$} & $\mathrm{PA}$ & -1978 & 2.75 & 17612.39 & 87.16 & Agrégation \\
\hline & $\mathrm{CJ}$ & 216 & 3.49 & 27442.66 & 304.89 & Création \\
\hline & SNA & -1105 & 0.82 & -753.39 & -72.27 & Suppression \\
\hline & $\mathrm{F}$ & -1193 & 0.55 & -14943.78 & -121.99 & Suppression \\
\hline & $\mathrm{S}$ & 66 & 0.90 & -24208.90 & 167.90 & Dissection \\
\hline
\end{tabular}

Légende $: \mathrm{PA}=$ Plantation arborée, $\mathrm{CJ}=$ Champs et Jachère, $\mathrm{SNA}=$ Sol nu et Agglomération, $\mathrm{F}=$ Forêt, $\mathrm{S}=$ Savane, $\mathrm{COS}=$ Classes d'occupation des sols, PTS = Processus de Transformation Spatiale. 
O. L. C. GBEDAHI et al. / Int. J. Biol. Chem. Sci. 13(1): 311-324, 2019

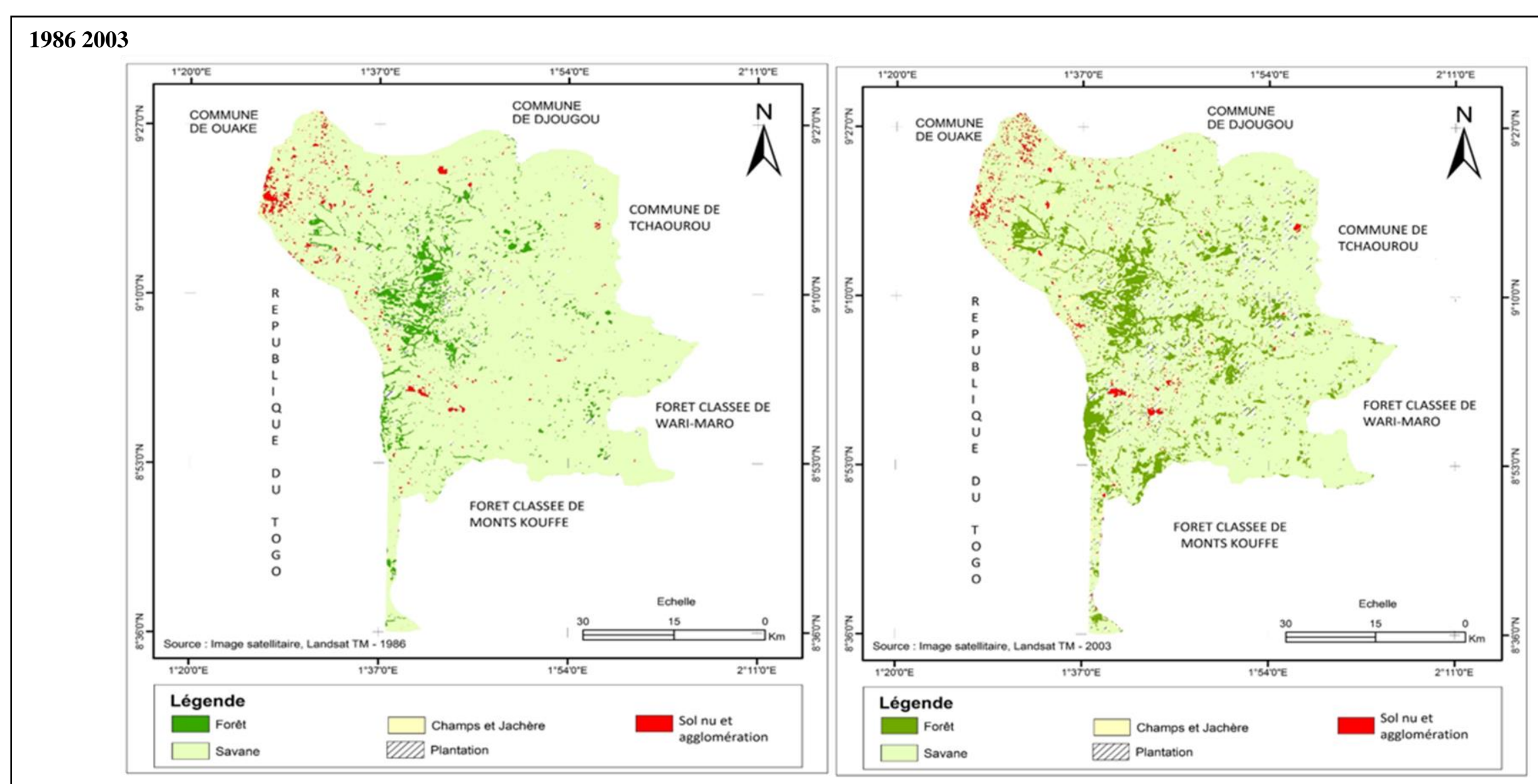

Réalisation : GBEDAHI Oméga Lydia C.

Figure 3: Dynamique de la structure spatiale entre 1986 et 2003 dans la zone d'intervention du PRRF, commune de Bassila, Bénin. 
O. L. C. GBEDAHI et al. / Int. J. Biol. Chem. Sci. 13(1): 311-324, 2019

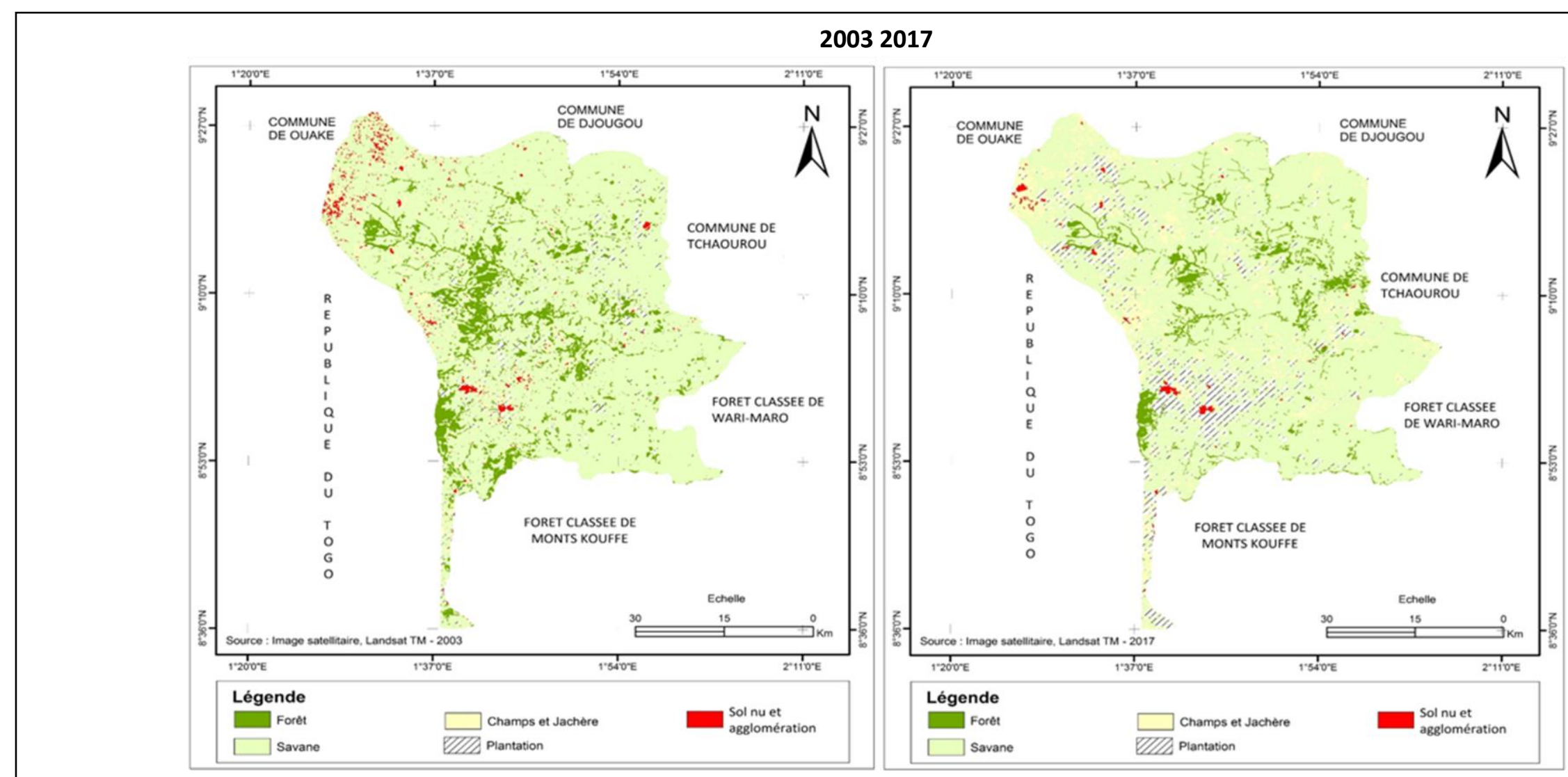

Réalisation : GBEDAHI Oméga Lydia C., Décembre 2016

Figure 4: Dynamique de la structure spatiale entre 2003 et 2017 dans la zone d'intervention du PRRF, commune de Bassila, Bénin. 


\section{DISCUSSION}

L'évolution de l'occupation des sols ces trente dernières années montre globalement une reforestation, quoi que faible, dans la zone d'intervention du PRRF dans la commune de Bassila. Cet accroissement des ressources forestières dans les zones d'intervention de projets d'aménagement forestiers a été aussi observé en Tanzanie (Blomley et al. 2008) et au Brésil (Melo et al., 2013). Contrairement à ces études, des études effectuées dans des zones non spécifiques à des projets forestiers telles que celles de Hountondji et al. (2008), Mama et al. (2014) et Issiaka et al. (2016) ont montré une dynamique spatio-temporelle régressive des ressources forestières en zone sahélienne et soudanienne de l'Afrique de l'Ouest. En effet, alors que Hountondji et al. (2008) ont montré une dégradation des ressources forestières en zone sahélienne, Mama et al. (2014) ont montré une déforestation drastique dans les communes de Bantè, Glazoué et Ouèssè dans la zone soudano-guinéenne au Bénin et Issiaka et al. (2016) ont démontré une déforestation au profit des savanes dans les parcours naturels de troupeaux transhumants dans les communes de Banikoara et de Karimama au Bénin. La reforestation remarquée dans la commune de Bassila pourrait donc être due à la mise en œuvre du PRRF dans cette commune. Néanmoins, le fait que la reforestation marquante observée pendant la période de mise en œuvre du projet soit atténuée par une destruction des forêts tout aussi marquante dans la période après projet montre que cet impact positif du PRRF n'est pas prolongé audelà de sa période d'intervention et qu'il existe des conditions après projet néfastes à la conservation des forêts dans la commune de Bassila. Ceci pourrait être dû à une absence de suivi des activités d'aménagement et de conservation dans les massifs forestiers aménagés (Brancalion et al., 2010). En effet, cette étude a révélé que, après la cessation du PRRF, le suivi des activités d'aménagement des forêts telles que l'entretien et la surveillance des plantations installées avec l'appui du projet, les campagnes de sensibilisation à la gestion durable des ressources forestières et la surveillance des massifs forestiers contre l'exploitation anarchique, a connu un relâchement. $\mathrm{Ce}$ relâchement pourrait donc avoir eu comme conséquence l'oubli, par les populations, des bonnes pratiques apprises. Ces dernières auraient alors renouées avec des habitudes destructrices de forêts telles que l'exploitation incontrôlée de bois, l'installation de champs dans les forêts, la carbonisation (Tente et al., 2011) et les feux de brousse incontrôlés (Gueguim et al., 2018). Il est donc nécessaire que soit maintenu, à la fin des projets forestiers, un minimum d'activités d'aménagement telles que l'entretien et la surveillance des plantations installées et la sensibilisation des populations à la gestion durable des ressources forestières, afin de ne pas laisser s'émousser l'engagement des populations riveraines à la conservation des ressources forestières. Par ailleurs, l'augmentation des superficies de plantations monospécifiques dans la commune de Bassila pendant et après le projet PRRF pourrait être attribuée à la mise en œuvre dudit projet dans cette région. Cette augmentation de plantations se faisant au détriment des formations végétales naturelles, l'on pourrait déduire que l'intervention du PRRF à Bassila bien qu'ayant conduit à une amélioration du couvert végétal, soit défavorable à la conservation de la biodiversité. Ce résultat confirme ceux de Voreux (2015) qui démontre qu'en matière de gestion durable des forêts tropicales, la conservation de la biodiversité est assimilée, dans la littérature, à la conservation du couvert forestier et de quelques espèces d'importance économique. Dans ce cas, il est très important qu'au cours de l'élaboration des plans d'aménagement et de gestion des forêts, la question de la conservation de la biodiversité soit réellement prise en compte afin que les objectifs assignés à l'aménagement forestier et les activités qui en découlent favorisent la conservation de la diversité biologique.

\section{Conclusion}

La mise en œuvre du PRRF dans la commune de Bassila s'est traduite, certes, par une restauration quoi que modeste des espaces 
forestiers, mais aussi par une certaine perte de la biodiversité forestière. En effet, les efforts déployés pendant la phase de mise en œuvre du projet qui ont entre-temps fait doubler les superficies de forêts dans la commune, ont pratiquement été réduits à néant après la fin du projet. On se retrouve alors, trente (30) années après le démarrage du PRRF et en ce qui concerne la couverture forestière dans la Commune de Bassila, pratiquement à la situation de départ de l'année 1988. La perte de la diversité biologique, quant à elle, s'est illustrée par la création et l'agrandissement de plantations monospécifiques au détriment de formations végétales naturelles. $\mathrm{La}$ progression des espaces de champs et jachères en défaveur des savanes, observée entre 1986 et 2017 assombrit davantage ce tableau. La réussite de l'intervention du projet PRRF à assurer la restauration et la gestion durable des ressources forestières de la commune de Bassila au Bénin est assez mitigée. Les causes probables de cette situation pourraient être le relâchement, voire l'abandon de la surveillance des massifs forestiers contre l'exploitation forestière anarchique après la fin du projet PRRF et le développement de plantations monospécifiques dans la zone d'intervention dudit projet. Un certain suivi de l'aménagement des massifs forestiers et un programme minimum de sensibilisation après la fin des projets forestiers de même qu'une meilleure prise en compte de la question de la conservation de la biodiversité dans l'aménagement et la gestion des forêts sont donc nécessaires pour une gestion durable de ces forêts.

\section{CONFLIT D'INTERETS}

Les auteurs déclarent n'avoir aucun conflit d'intérêts.

\section{CONTRIBUTIONS DES AUTEURS}

Chacun des auteurs, cités plus haut, a contribué de manière particulière à la réalisation de ce travail. Ainsi, OLCG en tant que premier auteur, a effectué, sous la supervision et la direction des autres auteurs, ce travail depuis la collecte des données jusqu'à la rédaction de ce article en passant par le traitement et l'analyse des données, l'interprétation et la discussion des résultats obtenus ; SSHB a contribué en apportant son expertise en ce qui concerne les questions d'écologie et d'étude de la dynamique du couvert végétal; AM a contribué à la réalisation de ce travail en apportant essentiellement son expertise en télédétection et Système d'Information Géographique (SIG) dans le cadre de l'approche cartographique utilisé ; GNG a contribué à ce travail en y apportant son expertise en Ecologie du paysage ; enfin NSY pour la réalisation de ce travail, a apporté son expertise dans les questions de conservation de la biodiversité et des autres services écosystémiques.

\section{REMERCIEMENTS}

Nos remerciements vont à l'endroit de tous ceux qui ont d'une manière ou d'une autre aidé à la réalisation de ce travail. De manière particulière, nous remercions le Directeur du Laboratoire d'Ecologie, de Botanique et de Biologie Végétale (LEB) de l'Université de Parakou, République du Bénin le Dr. Armand K. NATTA pour avoir mis à notre disposition au LEB, le cadre approprié et tous les moyens nécessaires à la réalisation de cette étude. Nous remercions aussi particulièrement l'administration forestière pour nous avoir facilité les travaux de terrains.

\section{REFERENCES}

Abdel Kawy WA, Belal AA. 2011. GIS to assess the environmental sensitivity for desertification in soil adjacent to ElManzala Lake, East of Nile Delta, Egypt. American-Eurasian J. Agric. \& Environ Sci., 10 : 844-856.

Adanguidi J. 2001. Réseaux, marchés et courtage. La filière igname au Bénin (1990-1997). Thèse de doctorat. Hamburg Lit Verlag 2001. xxiii-301p.

Atindogbé G. 2004. Analyse de la structure spatiale des arbres: Application à l'enrichissement des savanes boisées de la zone soudanienne au Bénin. Thèse d'Ingénieur Agronome, FSA/UAC, Bénin.

Avakoudjo J, Mama A, Toko I, Kindomihou V, Sinsin B. 2014. Dynamique de l'occupation du sol dans le Parc National 
du W et sa périphérie au Nord-Ouest du Bénin. Int. J. Biol. Chem. Sci., 8(6): 2608-2625.

DOI : http://dx.doi.org/10.4314/ijbcs.v8i6.22.

Baco MN, Tostain S, Mongbo RL, Biaou G, Lescure JP. 2007. Igname, plante alimentaire commerciale et culturelle au nord Bénin. Annales des sciences Agronomiques, 9(2). DOI: http://dx.doi.org/10.4314/asab.v9i2.432 51.

Barima YSS, Barbier N, Bamba I, Traore D, Lejoly J, Bogaert J. 2009. Dynamique paysagère en milieu de transition forêtsavane ivoirienne. BFT, 299(1): 15-25. DOI:

https://doi.org/10.19182/bft2009.299.a20 419

Blomley T, Pfliegner K, Isango J, Ahrends EZA, Burgess N. 2008. Seeing the wood for the trees: an assessment of the impact of participatory forest management on forest condition in Tanzania. Fauna \& Flora International, Oryx, 42(3): 380$391 \quad$ DOI: https://doi.org/10.1017/S0030605308071 433

Bogaert J, Ceulemans R, Salvador-Van Eysenrode D. 2004. Decision tree algorithm for detection of spatial processes in landscape transformation. Environ. Manage, 33 : 62-73. DOI: 10.1007/s00267-003-0027-0.

Bouko BS, Sinsin B, Soulé GB. 2007. Effets de la dynamique d'occupation du sol sur la structure et la diversité floristique des forêts claires et savanes au Bénin. Tropicultura, 25(4) : 221-227.

Brancalion PHS, Rodrigues RR, Gandolfi S, Kageyama PY, Nave AG, Gandara FB, Barbosa LM, Tabarelli M. 2010. Legal instruments can enhance high-diversity tropical forest restoration. Revista Árvore, 34(3): 455-470. DOI: http://dx.doi.org/10.1590/S010067622010000300010.

FAO. 2016. Situation des forêts du monde 2016. http://www.fao.org/3/a-i5850f.pdf (visité le 1.16.18).

Hincourt D. 1991. Carte pédologique et sa notice explicative. Bassila, Bénin p.51.
Houinato M, Sinsin B, Lejoly J. 2001. Impact des feux de brousse sur la dynamique des communautés végétales dans la forêt de Bassila (Bénin). Acta Bot. Gall., 148 : 237-251.

DOI: https://doi.org/10.1080/12538078.2001.1 0515891.

Hountondji YC. 2008. Dynamique environnementale en zones sahélienne et soudanienne de l'Afrique de l'Ouest: Analyse des modifications et évaluation de la dégradation du couvert végétal. Thèse de doctorat, Université de Liège, Liège, p.153.

IFN. 2007. Rapport d'exécution de l'Inventaire Forestier National (IFN), Projet Bois de Feu, phase II. Cotonou, Bénin, ministère de l'Environnement et de la Protection de la Nature, p.41.

IGN France. 1969. Carte de l'Afrique de l'Ouest à $1 / 200$ 000. République du Dahomey. Feuilles NC-31-II, NC-31-III, NB-31-XX-XXI.

INRAB. 2001. Recherche agricole pour le développement. Rapport annuel. Cotonou, Bénin, Institut national de recherche agronomique du Bénin, p.70.

INSAE. 2003. Troisième recensement général de la Population et de l'habitation. Synthèse des analyses en bref. Direction des études démographiques. Cotonou, octobre 2003, p.35.

INSAE. 2016. Effectif de la population des villages et quartiers de ville du Bénin (RGPH-4, 2013), p.83.

Issiaka NT, Arouna O, Imorou IT. 2016. Cartographie de la Dynamique spatiotemporelle des parcours naturels des troupeaux transhumants dans les communes de Banikoara et de Karimama au Benin (Afrique de l'Ouest). ESJ, 12(32): 1857-1881. DOI: http://dx.doi.org/10.19044/esj.2016 .v12n32p251.

Geist H, Lambin E. 2001. What drives tropical deforestation? A meta-analysis of proximate and underlying causes of deforestation based on subnational case study evidence. Land-Use and LandCover Change (LUCC) Project, International Geosphere-Biosphere 
Programme (IGBP), LUCC Report Series: 4, p.136.

Gueguim CD, Tchamba NM, Fotso CR. 2018. Dynamique spatio-temporelle des feux de brousse dans le Parc National du Mbam et Djerem (Cameroun). Int. J. Biol. Chem. Sci., 12(2): 728-748. DOI: https://dx.doi.org/10.4314/ijbcs.v12i2.10

Mama A, Bamba I, Sinsin B, Bogaert J, DeCannière C. 2014. Déforestation, savanisation et développement agricole des paysages de savanes-forêts dans la zone soudano-guinéenne du Bénin. BFT, 322 : 65-75.

DOI: https://doi.org/10.19182/bft2014.3 22.a31237.

Mas JF. 2000. Une revue des méthodes et des techniques de télédétection du changement. Can. J. Remote Sens., 26: 349-362.

DOI:

https://doi.org/10.1080/07038992.2000.1 0874785.

Mayaux P, Eva H, Fournier A, Sawadogo L, Palumbo I, Grégoire J-M. 2007. Apport des techniques spatiales pour la gestion des aires protégées en Afrique de l'Ouest. In Quelles Aires Protégées pour l'Afrique de l'Ouest? Conservation de la Biodiversité et Développement, Fournier A, Sinsin B, Mensah GA (éds). IRD, Colloques et Séminaires: Paris, France ; 321-328.

Melo FP, Pinto SR, Brancalion PH, Castro PS, Rodrigues RR, Aronson J, Tabarelli M. 2013. Priority setting for scaling-up tropical forest restoration projects: Early lessons from the Atlantic Forest Restoration. Pact. Environ. Sci. Policy, 33: 395-404.

Ngueguim JR, Chokona Gatchui H, Ayobami ST, Orimoogunje OO. 2009. Evaluation of logging impacts on tropical rainforest in Eastern Cameroon using Remote Sensing and GIS techniques. Int. J. Biol. Chem. Sci., 3(4): 771-785. DOI: https://dx.doi.org/10.4314/ijbcs.v3i4.471 63.

PRRF 2003. Aménagement des forêts privées dans la région de Bassila au Nord-Bénin. Projet de Restauration des Ressources Forestières de Bassila, Dossier technique, p.72.

PRRF 2004. Recueil de cartes. Projet de Restauration des Ressources Forestières de Bassila, Bénin, p.125.

Robin M. 2002. Télédétection, des satellites au SIG. Une analyse complète du processus de création d'un type essentiel d'information géographique. Nathan Université p.318.

Tente B, Baglo MA, Dossoumou JC, Yedomonhan H. 2011. Impacts des activités humaines sur les ressources forestières dans les terroirs villageois des communes de Glazoué et de DassaZoumè au centre-Bénin. Int. J. Biol. Chem. Sci., 5(5): 2022-2030. DOI : https://dx.doi.org/10.4314/ijbcs.v5i5.22.

Vermeulen C, Vandenhaute M, Dethier M, Ekodeck H, Nguenang GM, Delvingt W. 2006. De Kompia à Djolempoum : sur les sentiers tortueux de l'aménagement et de l'exploitation des forêts communautaires au Cameroun. VertigO - la revue électronique en sciences de l'environnement [En ligne], 7(1). DOI : $10.4000 /$ vertigo.2149.

Voreux C. 2015. La gestion durable des forêts tropicales. De l'analyse critique du concept à l'évaluation environnementale des dispositifs de gestion. Analyse d'ouvrage. BFT, 325(3): 3p. 